\title{
EXTRACONJUNCTIVAL APPROACH TO THE EXTRA-OCULAR MUSCLES*
}

BY

\author{
M. SARWAR \\ Oxford
}

SINCE contact lenses have been used for monocular conditions of impaired vision in which the eye is divergent, e.g., aphakia and corneal scars, it has been found that although vision could be improved the affected eye remained divergent.

Some of these eyes, i.e. those with small degrees of divergence, attained single binocular vision with orthoptic treatment; others with larger angles had to be helped surgically.

Orthodox trans-conjunctival surgery on these muscles was not very suitable for the following reasons:

(i) The majority of lenses which can be worn with comfort and without corneal haze require large haptic surfaces and need to be ventilated.

(ii) The haptic surfaces of these lenses extend beyond the sites of insertion of the muscles, and any scarring of this area naturally upsets the fit and balance of the lenses, rendering them unwearable.

(iii) In certain cases, although the lens might still be wearable, the haze-free time is shortened.

(iv) In yet other cases, the ventilating bubble increases in size owing to the disturbance in the fit, thus interfering with clear sight.

To overcome these difficulties an attempt was made to reduce the diameter of the lenses, but this was found to upset their balance. The contact lens in these cases has to be fitted before any operative intervention in order to obtain the full benefit of restored visual acuity and orthoptic treatment; the residual divergence only can then be treated surgically. The orthodox transconjunctival approach to the muscles in these cases would therefore mean scarring in the haptic area of the lens with the consequences described above. Refitting over a scarred area is the only alternative, and this is a technically difficult process.

Taking into consideration the various factors, I thought it would be best to approach the muscles away from their insertions to allow the scar tissue to form well away from the haptic area of the lens, and so devised the technique of extraconjunctival approach to the muscles, which has proved uniformly successful so far.

\section{Procedure}

Instruments.-As for a routine squint operation with a double-armed 000 catgut suture if not used normally and a Graefe knife.

Anaesthesia.-General.

*Received for publication December 3, 1953. 
Technique.-The lids may be infiltrated with saline and adrenaline with advantage.

\section{(1) External Rectus}

(a) The lids are held apart by the assistant and the eye turned inwards by a traction suture at the limbus, thus exposing the external canthus. An incision is now made as in Fig. $1(a)$ with a view to separating the conjunctiva from the outer ends of the upper and lower tarsal plates.

(b) This separation is continued with fine scissors backwards and towards the globe. This dissection exposes a mass of tissue consisting of the lateral check ligament, connective tissue, and the sheath of the external rectus muscle (Fig. 1b).

(c) The external check ligament is now cut (Fig. 1c).

(d) An incision is now made in the connective tissue and sheath in the direction of the muscle fibres. The connective tissue and sheath are reflected upwards and downwards and the muscle exposed. This procedure is necessary to avoid any chance of injury to the nerve to the inferior oblique which runs along the inferior border of the lateral rectus part of the way (Fig. 1d).

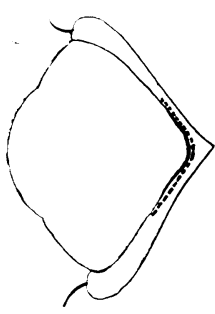

(a) Line of incision

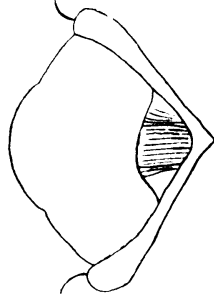

(b) Reflected conjunctiva

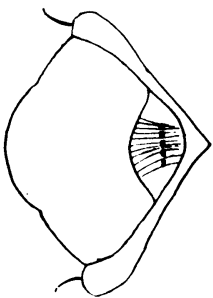

(c) Line of incision through check ligathrough check ligament
rectus

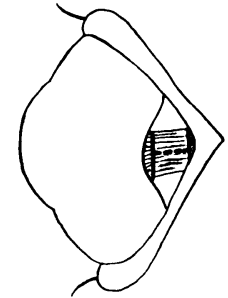

(d) Incision along length of muscle

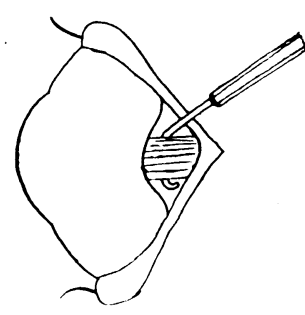

(e) Muscle on hook

FIG. 1.-External rectus muscle approach.

(e) The muscle is now picked up on a squint hook (Fig. 1e); its identity may be confirmed by pulling it laterally, when the eye should turn in the direction of action of the muscle and a small dimple should appear at the site of insertion of the muscle. It is necessary to be quite certain of the identity of the muscle as the inferior oblique is inserted in this region and may be picked up in error.

( $f$ ) If a resection is required it is carried out in the usual way. As a recession is not possible

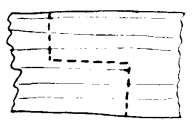

(a) Line of muscle section

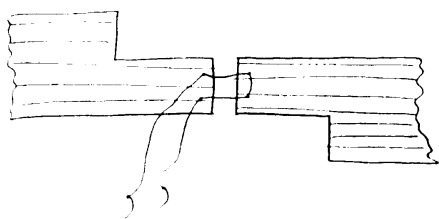

(b) Mattress suture joining ends of muscle

FIG. 2.-Lengthening of muscle. by this technique the muscle has to be lengthened. The muscle fibres are cut half-way across and then along for a distance slightly longer than half of the total lengthening required, again as in Fig. $2 a$. The two cut ends are then sutured together by means of a mattress suture as shown in Fig. $2 b$. The muscle is now replaced, the wound closed and the tissues allowed to fall back into position. Only one stitch is inserted and then across through the conjunctiva and the apex of the external canthus. No dressing is required.

\section{(2) Internal Rectus}

(a) The lids are held apart by means of a speculum and the eye turned outwards by a suture at the limbus. An incision is made along the lateral margin of the caruncle (Fig. $3 a$ ).

(b) The conjunctiva is dissected outwards from the skin of the caruncle. This dissection exposes the internal rectus muscle covered by its check ligament and sheath and surrounded by connective tissue (Fig. $3 b$ ).

(c) The muscle is picked up on a squint hook and cleared of its sheath and tissue and its identity confirmed as for the external rectus (Figs $3 c$ and $3 d$ ). 


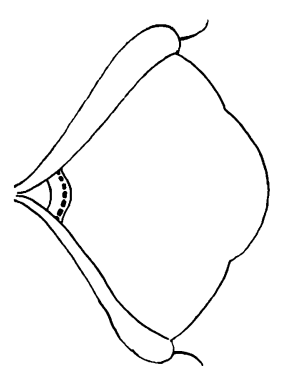

(a) Line of incision

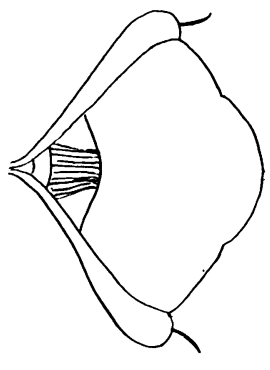

(b) Tissues and muscles exposed

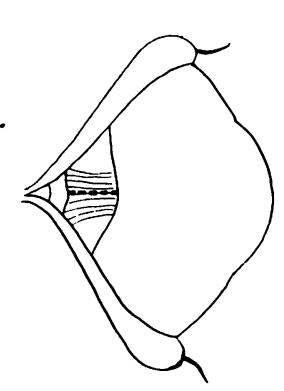

(c) Incision along muscle

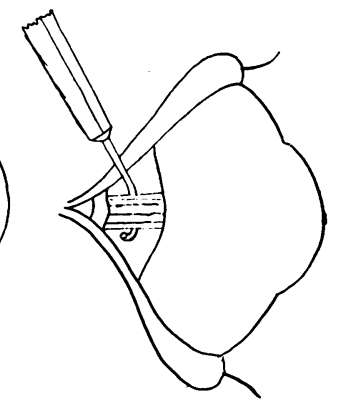

(d) Muscle on hook

FIG. 3.-Internal rectus muscle approach.

(d) The necessary resection or lengthening is carried out.

(e) The muscle is replaced, haemostasis achieved and the tissues smoothed back into position.

$(f)$ The conjunctiva is stitched back to the caruncle with one suture. No dressing is required.

Motility exercises should be started the next day, and the contact lens can be worn. Occasionally there is some conjunctival chemosis, usually caused by indifferent haemostasis at the end of the operation or by reaction to the catgut. This would naturally interfere with lens wearing, but can be controlled rapidly by using gutt. argyrol 5 per cent. with adrenaline. Motility exercises should be carried out whilst the eye is still oedematous, as they help to reduce the oedema. Orthoptic treatment should commence as soon as the lens can be worn.

For small divergent angles only a resection of the internal rectus is needed. A 4-mm. resection secures a correction of about $5-8^{\circ}$. For larger angles the lateral rectus has to be tackled as well-a 4-mm. resection of the internal rectus and an 8-mm. lengthening of the external rectus can correct an angle of $25-30^{\circ}$.

\section{Discussion}

As stated above this technique has been uniformly successful so far, and it was therefore thought advisable to compare it with the orthodox technique of extra-ocular surgery. An experiment was carried out on two cases through the courtesy of Mr. Hatfield Wright of Swindon. Bilateral muscle surgery had been decided on in these two cases, and at operation Mr. Wright did one eye using the orthodox technique whilst I did the other one using the extraconjunctival approach. No difference in time of healing, extent of mobility of the eye, or post-operative oedema or comfort of the children was observed. Apart from the site of the scar the result was identical. As the extraconjunctival method does not appear to offer any advantage over the transconjunctival tèchnique, and is certainly more difficult to carry out, I think that it should be used only in contact lens cases or special circumstances of like nature.

I wish to record my appreciation of Mr. Hatfield Wright's generosity in providing patients and hospital facilities for the experiment. My thanks are due to my technician Geoffrey Rhodes for the drawings. 